\title{
Zinc tin oxide thin film transistors produced by a high rate reactive sputtering: effect of tin composition and annealing temperatures
}

\author{
Kham M. Niang ${ }^{*}, 1$, Junhee Cho ${ }^{1}$, Aditya Sadhanala ${ }^{2}$, William I. Milne', Richard H. Friend ${ }^{2}$ and Andrew J. \\ Flewitt ${ }^{1}$ \\ ${ }^{1}$ Electrical Engineering Division, Department of Engineering, University of Cambridge, 9 JJ Thomson Avenue, Cambridge, CB3 \\ OFA, United Kingdom \\ ${ }^{2}$ Department of Physics, University of Cambridge, 19 JJ Thomson Avenue, Cambridge, CB3 0HE, United Kingdom
}

Received ZZZ, revised ZZZ, accepted ZZZ

Published online ZZZ (Dates will be provided by the publisher.)

Keywords TFTs, oxides, indium free, sputtering

* Corresponding author: e-mail kmn36@cam.ac.uk, Phone: +44 (0)1223 748305, Fax: +44 (0)1223 748348

Amorphous zinc tin oxides (a-ZTO) which are stoichiometrically close to the $\mathrm{Zn}_{2} \mathrm{SnO}_{4}$ and $\mathrm{ZnSnO}_{3}$ phases have been deposited using remote-plasma reactive sputtering, and are incorporated as the channel layers in thin film transistors (TFTs). The influence of tin composition and annealing temperatures on the structural and phase evolutions of the thin films and the electrical performances of the TFTs are investigated. $\mathrm{Zn}_{2} \mathrm{SnO}_{4}$ exhibited randomly oriented polycrystalline peaks at annealing temperatures $\geq 700{ }^{\circ} \mathrm{C}$, while $\mathrm{ZnSnO}_{3}$ decomposed into $\mathrm{Zn}_{2} \mathrm{SnO}_{4}$ and $\mathrm{SnO}_{2}$ at $950{ }^{\circ} \mathrm{C}$. TFTs employing a $\mathrm{Zn}_{2} \mathrm{SnO}_{4}$ channel, after a post-deposition annealing at $500{ }^{\circ} \mathrm{C}$, exhibited a field effect mobility $\sim 14$ $\mathrm{cm}^{2} \mathrm{~V}^{-1} \mathrm{~s}^{-1}$ and a sub-threshold slope $\sim 0.6 \mathrm{~V} \mathrm{dec}^{-1}$. When the tin content is increased in the channel, as in $\mathrm{ZnSnO}_{3}$, TFTs exhibited an increase in field effect mobility $\sim 20$ $\mathrm{cm}^{2} \mathrm{~V}^{-1} \mathrm{~s}^{-1}$ but with a slight deterioration of sub-threshold slope to $\sim 0.8 \mathrm{~V} \mathrm{dec}^{-1}$. When the post-deposition annealing temperature is reduced to $300{ }^{\circ} \mathrm{C}$, a mobility as high as $10 \mathrm{~cm}^{2} \mathrm{~V}^{-1} \mathrm{~s}^{-1}$ is still achieved, however a significant shoulder in the $I_{D S^{-}} V_{G S}$ curve together with a higher offstate current is observed. TFT characteristics are explained by the sub-bandgap defect states measured by photothermal deflection spectroscopy and the extracted Urbach energies.
1 Introduction Ionic oxide semiconductors are important materials for large area electronic displays due to their high carrier mobility, transparency, and the possibility of processing at low temperatures. Indium gallium zinc oxide (IGZO) TFTs show carrier mobility of $\sim 10 \mathrm{~cm}^{2} \mathrm{~V}^{-1} \mathrm{~s}^{-1}$, which is an order of magnitude higher than that of hydrogenated amorphous silicon (a-Si:H) [1]. However, this material system is rather complex with three metal cations, two of which are expensive and resourcescarce elements. Given that a wide variety of oxides are available, research into simpler and less expensive material systems are vitally important from an economic point of view. Amorphous zinc tin oxide (a-ZTO) fits such a system and it has been previously reported that a-ZTO TFTs show high electron mobility [2]. Recent studies on a-
ZTO as a replacement for indium tin oxide anodes in OLED displays [3], the buffer layer in oxide solar cells [4], and the active layer in sensors [5] have clearly demonstrated the technological importance and versatility of this material for various applications.

Most oxides are produced by r.f. magnetron sputtering from ceramic targets, but the growth rate is very modest (a few $\mathrm{nm} \mathrm{min}^{-1}$ ) and the films tend to be highly stressed [6]. Reactive sputtering generally has a higher deposition rate, and allows the use of metal targets which are more durable and cheaper than ceramic targets, thus lowering production costs [7]. However, reactive sputtering is not widely utilized for the development of a-ZTO TFTs; this is the motivation for this work. 
In this report, a-ZTO thin films are reactively sputtered from zinc:tin alloy targets with Sn compositions of 10, 33 and 50 at.\%, and an optimized oxygen flow. Preferential sputtering results in the tin composition being higher in the films than in the targets. We have first systematically investigated the crystallographic properties and stoichiometry of thin films after annealing at temperatures up to $950{ }^{\circ} \mathrm{C}$ and characterised these by X-ray diffraction (XRD) and scanning electron microscopy (SEM). Films deposited from 10 and 33 at.\% targets are found to have tin compositions of 33 and 50 at.\% respectively, resulting in stoichiometries of the two common crystalline phases of ZTO: inverse spinel $\mathrm{Zn}_{2} \mathrm{SnO}_{4}$ and ilmenite $\mathrm{ZnSnO}_{3}$. These films have been successfully incorporated as channel layers in TFTs. Best performing TFTs consistently show field effect mobility as high as $20 \mathrm{~cm}^{2} \mathrm{~V}^{-1} \mathrm{~s}^{-1}$, switching ratio of $\sim 10^{8}$ and sub-threshold slope $\sim 0.7 \mathrm{Vdec}^{-1}$, which are obtained with a post-deposition annealing at $500{ }^{\circ} \mathrm{C}$. TFTs incorporating a-ZTO channel layer with lower annealing temperature are also reported. The effect of tin compositions and annealing temperatures on TFT performances are explained by the measured sub-bandgap defect states and Urbach energy.

2 Experimental details a-ZTO thin films were deposited without intentional substrate heating onto various substrates from the $\mathrm{Zn}: \mathrm{Sn}$ metal alloy targets with atomic tin contents of 10,33 and $50 \%$. As references, $\mathrm{ZnO}$ and $\mathrm{SnO}_{2}$ thin films are also sputtered from pure metal targets. The chamber pressure during sputtering was $\sim 6$ $\times 10^{-3}$ mbar which was achieved with argon and oxygen flows of $60 \mathrm{sccm}$ and $35 \mathrm{sccm}$ respectively. An RF launch power of $800 \mathrm{~W}$ and a target bias power of $500 \mathrm{~W}$ were used. The details of the sputtering system can be found elsewhere [8]. A-ZTO and $\mathrm{ZnO}$ layers which are used for film characterisations are $\sim 250$ to $350 \mathrm{~nm}$ thick and those used for TFT channel layers are $\sim 50 \mathrm{~nm}$ thick. Postdeposition annealing of the films was performed at temperatures between 300 and $950{ }^{\circ} \mathrm{C}$ in an oven in air. The annealing time was 1 hour, except for two samples annealed at $950{ }^{\circ} \mathrm{C}$ (ZTO50 and ZTO65) where the annealing time was reduced to 45 minutes.

The film thickness was determined using a Gaertner $\mathrm{He}-\mathrm{Ne}(633 \mathrm{~nm})$ ellipsometer and Veeco Dektak profilometer. The crystallinity of the films was determined by a Phillips PW 1820 XRD using a $\mathrm{Cu}-\mathrm{K}_{\alpha}$ radiation and a monochromator with the divergence slit and receiving slit setting of $0.5 \mathrm{~mm}$ and $0.2 \mathrm{~mm}$ respectively. SEM images and estimation of chemical composition were performed on a Leo Gemini 1530VP FEG SEM/EDX system. A more accurate ThermoScientific Multilab-2000 X-ray photoelectron spectroscopy was also used on some selected films. The electronic density of states within the bandgap was examined using photothermal deflection spectroscopy (PDS), which is a very sensitive optical absorption technique [9].
Bottom gate, inverted staggered structure TFTs were fabricated using thermally-grown $\mathrm{SiO}_{2}$ films of $200 \mathrm{~nm}$ thickness as the gate dielectric and heavily-doped p-type $\mathrm{Si}$ (100) substrates as the gate electrode. Thermally evaporated aluminium $(\sim 270 \mathrm{~nm})$ was used as the source and drain contacts. The active layer and the source/drain contacts were deposited and patterned using conventional photolithography and lift off methods after annealing. The TFTs with channel width to lengh ratio of $50(W / L=1000$ $\mu \mathrm{m} \mathrm{/} 20 \mu \mathrm{m}$ ) were measured in the dark, at room temperature using a Wentworth probe station inside a Faraday cage with a HP4140B dual voltage source picoammeter.

\section{Results and Discussion}

3.1 Thin film characterisations Figure 1 shows the deposition rate as a function of tin composition in the films $(\mathrm{Sn} /(\mathrm{Zn}+\mathrm{Sn})$ in atomic \%). The deposition rate is the lowest for pure $\mathrm{ZnO}\left(\sim 12 \mathrm{~nm} \mathrm{~min}{ }^{-1}\right)$ and is the highest for pure $\mathrm{SnO}_{x}\left(\sim 34 \mathrm{~nm} \min ^{-1}\right)$. The deposition rate of ZTO varies between 15 to $22 \mathrm{~nm} \mathrm{~min}^{-1}$ and it increases monotonically with the tin composition in the film.

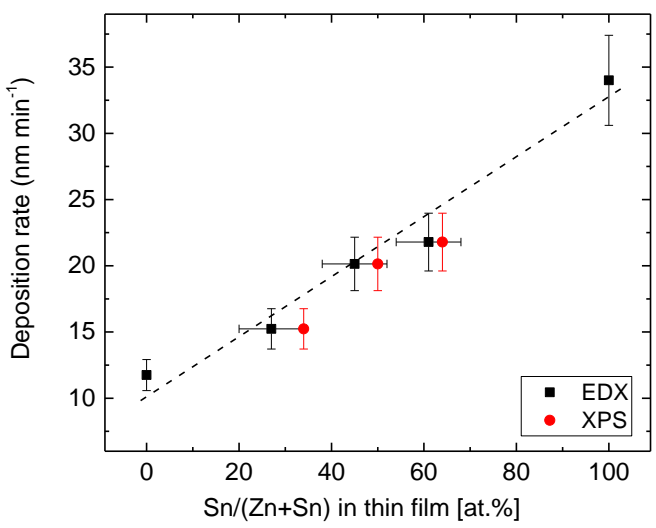

Figure 1 Deposition rate as a function of tin in the ZTO thin films. A dashed line is drawn as a guide to the eye.

The tin composition in ZTO is determined by EDX on most of the films and by XPS on selected films. It is found that the ZTO film which was sputtered from a zinc:tin alloy target with $10 \% \mathrm{Sn}$ actually contains $\sim 33 \% \mathrm{Sn}$. Similarly, ZTO films sputtered from a zinc:tin alloy target with $33 \%$ and $50 \%$ Sn contain $\sim 50 \%$ and $\sim 65 \%$ Sn respectively. Referring to the more accurate XPS data in Figure 1, the increase of tin in the films compared with the target is found to be a factor of 1.3 to 3.3. This is due to preferential sputtering which is commonly encountered in sputtering from a target with two or more elements [10]. It is also observed in rf magnetron sputtering but to a lesser degree, by a factor of 1.3 only [11-13]. In reactive sputtering, accurate flow of oxygen would be necessary to produce an oxide 
with the correct stoichiometry. Moreover, the deposition rate is also generally higher when sputtering from a metal target than a ceramic target. Therefore, it is not surprising that preferential sputtering is more pronounced in reactive sputtering from a metal than in magnetron sputtering from a ceramic.

From here on, the ZTO films will be referred to as ZTO33, ZTO50 and ZTO65 according to their tin composition in the film determined by XPS. The stoichiometry of the ZTO33 and ZTO50 are very close to the stoichiometry of $\mathrm{Zn}_{2} \mathrm{SnO}_{4}$ and $\mathrm{ZnSnO}_{3}$ respectively, which are the two common ZTO crystals.

Figure 2 shows the X-ray diffractogram of ZTO33, ZTO50 and ZTO65 films as-deposited and after annealing at various temperatures. As shown in Figure 2a, the asdeposited ZTO33 show diffraction peaks relating to those of the silicon substrate only, thus indicating that the film is likely to be amorphous. The film still appears to be amorphous after annealing at $500{ }^{\circ} \mathrm{C}$. Small peaks appear at $2 \theta=17.9,29.3,34.6$ and $42.1^{\circ}$ after annealing at $700{ }^{\circ} \mathrm{C}$ and their intensities increase after annealing at $900{ }^{\circ} \mathrm{C}$, along with the appearance of new smaller peaks at $36.1,44.5,52.1$ and $60.4^{\circ}$. These peaks match exactly with the power diffraction patterns of $\mathrm{Zn}_{2} \mathrm{SnO}_{4}$. Therefore, it is confirmed that ZTO33 deposited by reactive sputtering is indeed stoichiometrically $\mathrm{Zn}_{2} \mathrm{SnO}_{4}$.

Our results are consistent with previously reported $\mathrm{Zn}_{2} \mathrm{SnO}_{4}$ using rf magnetron sputtering from ceramic targets, and are also very similar to the temperature range at which recrystalliztion begins. Randomly oriented polycrystalline structured $\mathrm{Zn}_{2} \mathrm{SnO}_{4}$ has been reported at $660{ }^{\circ} \mathrm{C}$ by Young et al. [12], and at $750{ }^{\circ} \mathrm{C}$ by Satoh et al. [11]. Interestingly films with (111) only orientation are also reported in the latter paper when sputtering with oxygen and argon gas mixtures [11]. When spray pyrolysis was used, a thermal treatment in air at $1000{ }^{\circ} \mathrm{C}$ up to 10 hours is needed to obtain the polycrystalline $\mathrm{Zn}_{2} \mathrm{SnO}_{4}$ phase [14].

As shown in Figure 2b, the ZTO50 film is amorphous both as-deposited and after annealing at $500{ }^{\circ} \mathrm{C}$, similar to ZTO33 in Figure 2a. However, unlike ZTO33, the ZTO50 film still remains amorphous even after annealing at $700{ }^{\circ} \mathrm{C}$. After annealing at $950{ }^{\circ} \mathrm{C}$, diffraction peaks relating to $\mathrm{Zn}_{2} \mathrm{SnO}_{4}$ appear. Additionally, new peaks at $2 \theta=$ 26.8, 38.1 and $52^{\circ}$ also appear. These new peaks match the (110), (200) and (211) peaks of $\mathrm{SnO}_{2}$. Based on its stoichiometry, the ZTO50 should have exhibited peaks relating to $\mathrm{ZnSnO}_{3}$ after annealing, but that is not the case. Instead, peaks that are related to both $\mathrm{Zn}_{2} \mathrm{SnO}_{4}$ and $\mathrm{SnO}_{2}$ are observed. It has been previously reported that the $\mathrm{ZnSnO}_{3}$ is decomposed into $\mathrm{Zn}_{2} \mathrm{SnO}_{4}$ and $\mathrm{SnO}_{2}$ at temperatures between 700 to $800{ }^{\circ} \mathrm{C}$ [15]. That is in the same temperature range when ZTO50 recrystallizes and changes phase. It is also noted that the intensity of $\mathrm{SnO}_{2}$ peaks are higher than the $\mathrm{Zn}_{2} \mathrm{SnO}_{4}$ peak.
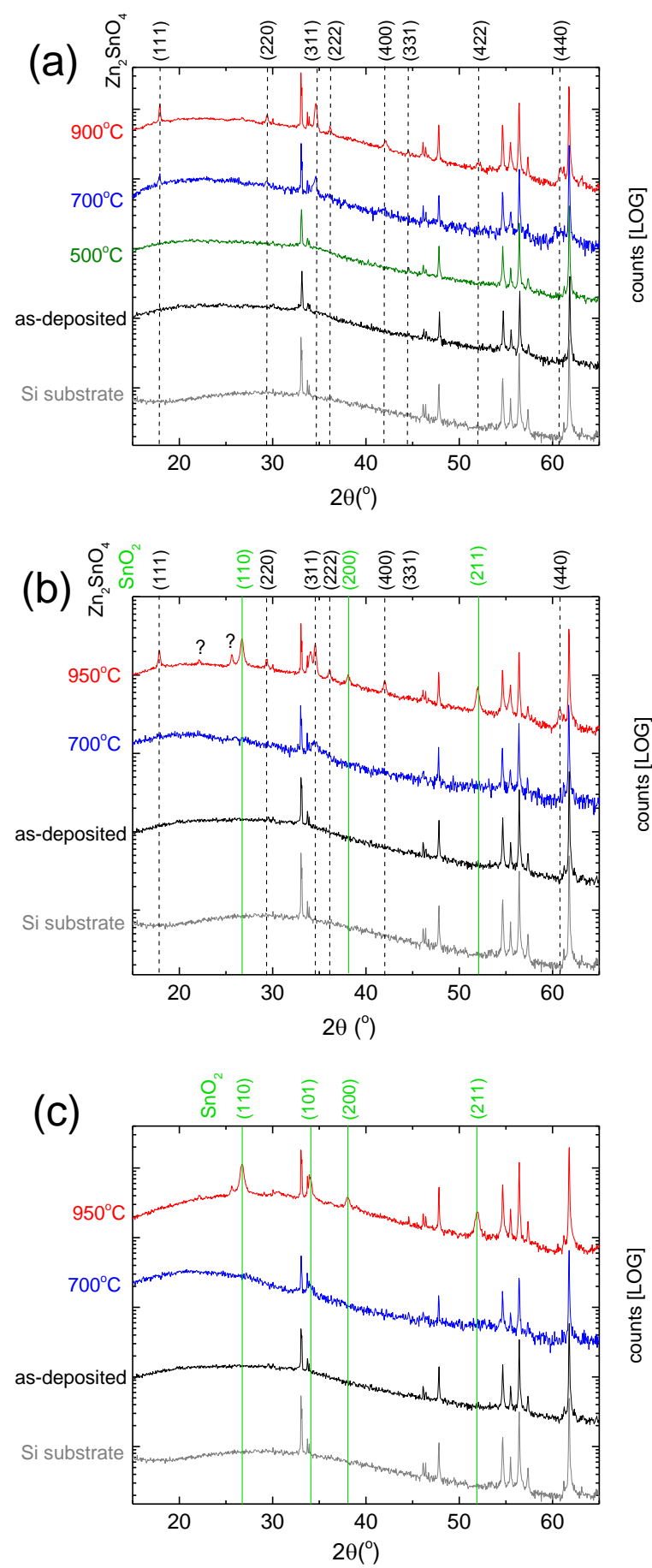

Figure 2 X-ray diffractogram of ZTO33, ZTO50 and ZTO65 (a-c) films as-deposited and after annealing at various temperatures. XRD of silicon is also shown to highlight substrate related peaks. (?) indicates peaks which are unidentified. Diffraction peaks of $\mathrm{Zn}_{2} \mathrm{SnO}_{4}$ (JCPDF 24-1470) are shown as dashed lines and those of $\mathrm{SnO}_{2}$ (JCPDF 44-1445) are shown as solid lines. 
Finally, XRD scans for ZTO65 are shown in Figure 2c. Similar to ZTO50 in Figure 2b, the ZTO65 remains amorphous after annealing up to $700^{\circ} \mathrm{C}$. After annealing at $950{ }^{\circ} \mathrm{C}$, only peaks relating to $\mathrm{SnO}_{2}$ appear but none relating to $\mathrm{Zn}_{2} \mathrm{SnO}_{4}$. It is also noteworthy that the intensities of the $\mathrm{SnO}_{2}$ peaks are equally as high in ZTO65 as in ZTO50. At this point, ZTO65 looks very similar to pure $\mathrm{SnO}_{2}$ sputtered using dc magnetron sputtering at $250{ }^{\circ} \mathrm{C}[16]$ or that produced by plasma enhanced chemical vapor deposition at $250{ }^{\circ} \mathrm{C}$ [17]. The chemical compositions of ZTO65 was not checked on this annealed sample, but it is very likely that zinc ablation has occurred [18]. In fact, when zinc stannate was prepared by spray pyrolysis, precursors with a zinc:tin ratio of 4:1 were needed to account for the loss by evaporation of zinc oxide during a very long heat treatment at $1000{ }^{\circ} \mathrm{C}$ [14].

SEM is used to investigate the surface morphology of ZTO films after annealing at temperatures $\geq 700{ }^{\circ} \mathrm{C}$. In Figure 3a, the top view SEM micrograph of ZTO33 after annealing at $700{ }^{\circ} \mathrm{C}$ shows a smooth surface with small but distinctive round grains. The SEM image after $500{ }^{\circ} \mathrm{C}$ (not shown) shows a similarly smooth surface, but with fewer round grains. Since XRD in Figure 2a indicates a predominantly amorphous film at $500{ }^{\circ} \mathrm{C}$ and small polycrystalline peaks at $700{ }^{\circ} \mathrm{C}$, the round grains indicate some local, short range ordering of crystals. After annealing at $900{ }^{\circ} \mathrm{C}$ (Figure $3 \mathrm{~b}$ ), the ZTO33 film shows irregular grains with large variation in sizes between 50 to $600 \mathrm{~nm}$. This is reflected in the higher intensity of the XRD peaks (Figure 2a). It is difficult to compare the microstructures of $\mathrm{Zn}_{2} \mathrm{SnO}_{4}$ between samples which were prepared by different methods, nonetheless SEM images of relatively homogenous, irregularly shaped grains or agglomerates have been reported by various groups $[14,19]$.
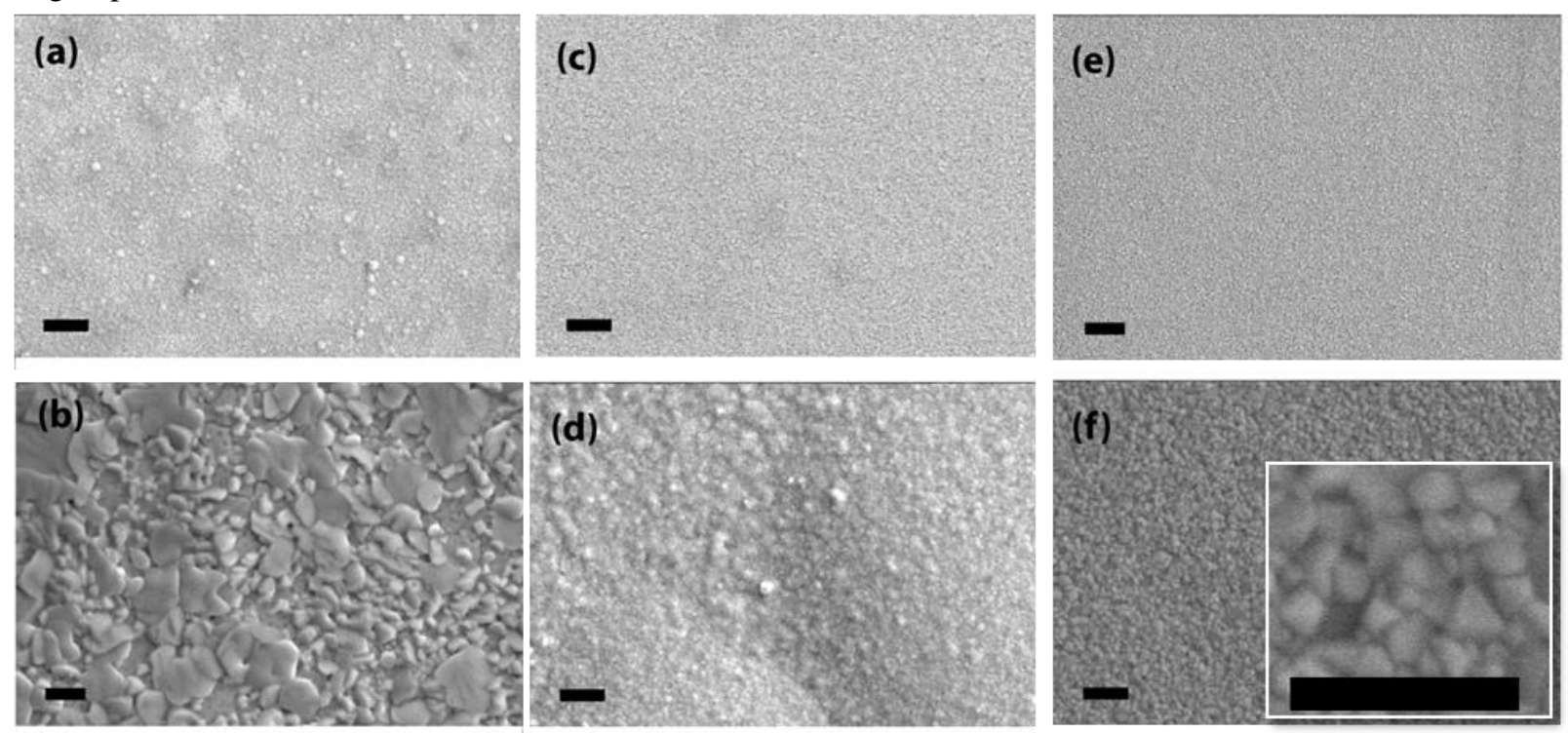

Figure 3 Top view scanning electron micrographs of ZTO thin films. In the top row are the ZTO33, ZTO50 and ZTO65 after annealing at $700{ }^{\circ} \mathrm{C}$ (a, c, and e). In the bottom row are the ZTO33 after annealing at $900{ }^{\circ} \mathrm{C} \mathrm{(b),} \mathrm{ZTO50} \mathrm{and} \mathrm{ZTO65} \mathrm{after} \mathrm{annealing} \mathrm{at}$ $950^{\circ} \mathrm{C}(\mathrm{d}$ and $\mathrm{f})$ respectively. Inset of figure $3 \mathrm{f}$ is a zoom-in of the grains. The scale bars are $400 \mathrm{~nm}$. 
It should be noted that the ZTO33 and ZTO50 films that are used as the channel layer in TFTs are annealed at temperatures $\leq 500{ }^{\circ} \mathrm{C}$, thus they would be still amorphous. They may look very similar having smooth, highly uniform surfaces, but they are very different materials and are stoichiometrically very close to $\mathrm{Zn}_{2} \mathrm{SnO}_{4}$ and $\mathrm{ZnSnO}_{3}$ respectively.

\subsection{Thin film transistors}

3.2.1 Effect of tin compositions Figure 4 shows an overlay of the gate transfer characteristics for the $\mathrm{ZnO}$, ZTO33, ZTO50 and ZTO65 TFTs. The field effect mobility, $\mu_{F E}$, is calculated from,

$$
\mu_{F E}=\frac{\left[\partial I_{D S} / \partial V_{G S}\right]}{C_{o x}(W / L) V_{D S}}
$$

where $\partial I_{D S} / \partial V_{G S}$ is the transconductance, $C_{o x}$ is the oxide capacitance, and $W / L$ is the width to length ratio of the channel. The ZnO TFT exhibited a $\mu_{F E}$ of $0.7 \mathrm{~cm}^{2} \mathrm{~V}^{-1} \mathrm{~s}^{-1}$, a sub-threshold slope $\sim 4 \mathrm{Vdec}^{-1}$ and a switching ratio of $10^{6}$. A significant improvement in performance is observed in the ZTO33 TFT, exhibiting $\mu_{F E}$ of $13.5 \mathrm{~cm}^{2} \mathrm{~V}^{-1} \mathrm{~s}^{-1}$, a threshold voltage $\sim 10 \mathrm{~V}$, sub-threshold slope $\sim 0.55 \mathrm{~V}$ $\mathrm{dec}^{-1}$ and switching ratio of $5 \times 10^{8}$. A further increase in $\mu_{F E}\left(20.2 \mathrm{~cm}^{2} \mathrm{~V}^{-1} \mathrm{~s}^{-1}\right)$ is observed in ZTO50 with threshold voltage $\sim 8.3 \mathrm{~V}$, sub-threshold slope $\sim 0.78 \mathrm{~V} \mathrm{dec}^{-1}$ and a switching ratio of $5 \times 10^{8}$. It is noteworthy that the hysteresis is very small in our devices. Finally, the ZTO65 TFT exhibited no transistor characteristics and it could no long-

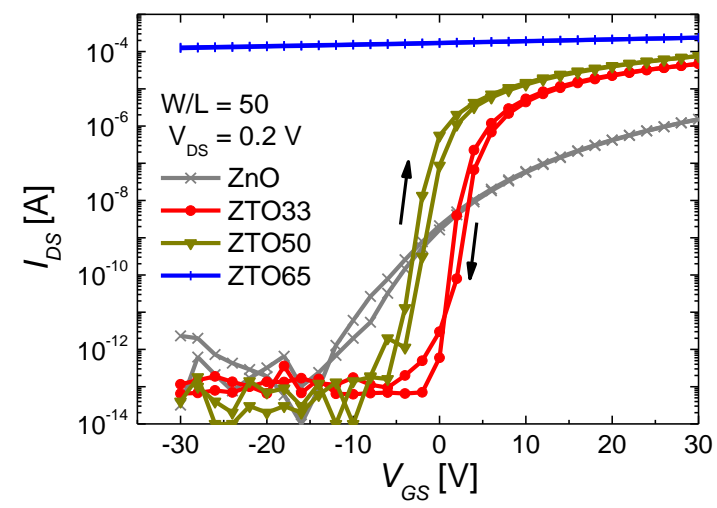

Figure 4 Gate transfer characteristics for the ZTO and ZnO TFTs measured with $V_{D S}=0.2 \mathrm{~V}$. A post-deposition annealing of the channel layer is performed at $500^{\circ} \mathrm{C}$ for 1 hour in air. er be used as a semiconductor channel. The TFT parameters are summarised in Table 1 .

It is well established that the performance of $\mathrm{ZnO}$ TFTs is greatly limited by the grain boundaries of the polycrystalline $\mathrm{ZnO}$ channel [21].

In Figure 4, a sharp increase of field effect mobility in $\mathrm{ZnO}$ to ZTO33, and a further increase to ZTO50 can be attributed to (i) the lack of grain boundaries in the channel layer arising from the amorphous nature of multicomponent oxides, and (ii) the effect of metal cation incorporation, as in this case, tin with its valency $(+2$ or +4$)$ will contribute more free carriers [1]. Another advantage of incorporation of heavy metal cations is the suppression of oxygen vacancy formation, which is attributed to their higher bond strength over $\mathrm{ZnO}$. Suppression of oxygen vacancies then enables a very low off state current [22]. Such a scheme has been employed in material systems other than zinc oxide; in this case, silicon dioxide is incorporated into indium oxide based TFTs [23].

However, the increase in carrier concentration and thus mobility in ZTO50 comes at the expense of higher subthreshold slope as it is increased from 0.55 to $0.78 \mathrm{~V} \mathrm{dec}^{-1}$. As the carrier concentration is further increased to $\sim 65 \%$ as shown in ZTO65, channel depletion can no longer be achieved.

It is well established that TFT performances are strongly affected by defect tail states and sub-bandgap states originating from structural disorder and defects [24]. These states are measured by PDS which is widely utilized in various applications [25,26]. Figure 5 shows the absorption spectra as a function of sub-bandgap energies $(\leq 3.2$ $\mathrm{eV}$ ) for the three ZTO films annealed at $500{ }^{\circ} \mathrm{C}$, which is the same anneal temperature as for the channel layers.

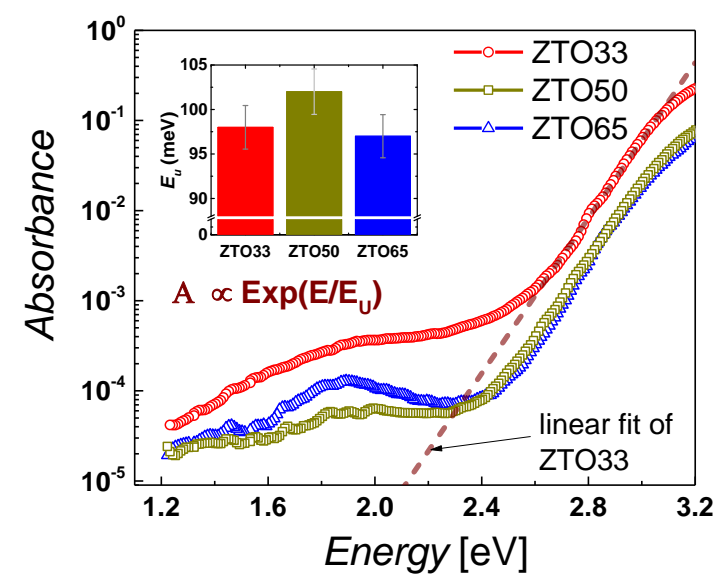

Figure 5 Absorption spectra for ZTO thin films annealed at $500{ }^{\circ} \mathrm{C}$ obtained using PDS. Inset shows the corresponding Urbach energy extracted by fitting the band-tail. 


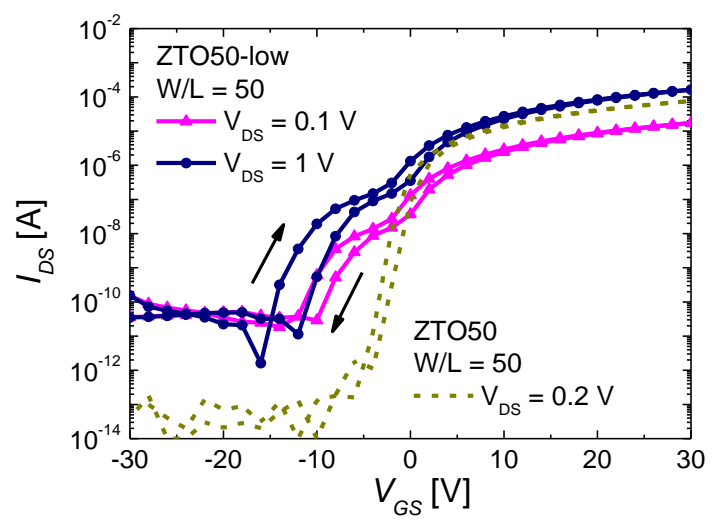

Figure 6 Gate transfer characteristics for the ZTO50-low TFTs measured with $V_{D S}=0.1$ and $1 \mathrm{~V}$. The channel layer is annealed at $300^{\circ} \mathrm{C}$ for 1 hour in air. For comparison the ZTO50 TFT is also shown.

The extracted Urbach energy, $E_{U}$, for ZTO33, ZTO50 and ZT65 are very similar: 98, 102 and $97 \mathrm{meV}$ respectively. These values are lower than the previously reported Urbach energies for ZTO $(110 \mathrm{meV})$ and IGZO $(110 \sim 160$ $\mathrm{meV}$ ) $[22,27,28]$. A very low Urbach energy $\sim 22 \mathrm{meV}$ has also been reported for IGZO [29]. It should be noted that different measurement techniques were used in all these papers. Since lower Urbach energy means fewer defect states in the bandgap, we can say that our ZTO films are of very good quality, which is also indicated by the device performance. It is generally accepted that the defect states detected by PDS are dominated by those at the valence band edge [27], which agrees well with the ZTO65 result in that it is not switching off because of excess carriers in the conduction band tails and not because of higher defects in the valence band tails.

Also shown in Figure 5 is the absorbance deep in the sub-bandgap $(1.2 \mathrm{eV}<\mathrm{E}<2.2 \mathrm{eV})$ and it is the highest in ZTO33, followed by ZTO65 and then ZTO50. The differences in the absorbance levels between samples are large, but there is no strong correlation observed between absorbance (deep defect states) and tin concentrations in aZTO.

3.2.2 Effect of annealing temperatures TFTs are fabricated employing ZTO50 with a lower post-deposition annealing at $300{ }^{\circ} \mathrm{C}$ (denoted as ZTO50-low) as shown in Figure 6. With a $300{ }^{\circ} \mathrm{C}$ anneal, TFT characteristics are observed but with a higher off-state current, $I_{\text {OFF }} \sim 10^{-10} \mathrm{~A}$ and a significant shoulder around $V_{G S}=0 \mathrm{~V}$. Compare to ZTO50 which is turned on at $V_{G S} \sim-5 \mathrm{~V}$, the ZTO50-low turned on at $V_{G S} \sim-15 \mathrm{~V}$. The device turn-on voltage is also sensitive to the applied $V_{D S}$. In the accumulation region, an order of magnitude increase in the $I_{D S}$ is observed as $V_{D S}$ is increased from 0.1 to $1 \mathrm{~V}$. Despite being less than ideal

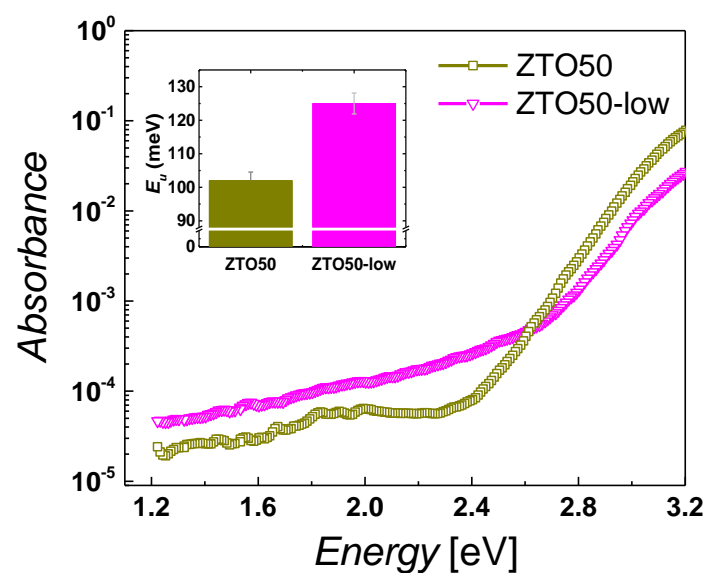

Figure 7 PDS absorption spectra for ZTO thin films annealed at either $300{ }^{\circ} \mathrm{C}$ (ZTO50-low) or $500{ }^{\circ} \mathrm{C}$ (ZTO50). Inset shows the corresponding Urbach energy extracted by fitting the bandtail.

devices, a high $\mu_{F E} \sim 10 \mathrm{~cm}^{2} \mathrm{~V}^{-1} \mathrm{~s}^{-1}$ is still achieved in ZTO50-low TFTs. Device parameters are also summarised in Table 1.

Table 1 Transistor parameters of ZnO and ZTO TFTs with a channel $W / L$ ratio of 50 , measured with a $V_{D S}$ of 0.1 or $0.2 \mathrm{~V}$ (as shown in Figure. 4 and 6).

\begin{tabular}{lcccc}
\hline \multicolumn{1}{c}{ TFTs } & $\begin{array}{c}V_{\text {th }} \\
{[\mathrm{V}]}\end{array}$ & $\begin{array}{c}\mu_{F E} \\
{\left[\mathrm{~cm}^{2} \mathrm{~V}^{-1} \mathrm{~s}^{-1}\right]}\end{array}$ & $\begin{array}{c}\text { S.S } \\
{\left[\mathrm{Vdec}^{-1}\right]}\end{array}$ & ION/IOFF \\
\hline ZnO & 17.6 & 0.7 & 4.0 & $10^{6}$ \\
ZTO33 & 10.0 & 13.5 & 0.55 & $5 \times 10^{8}$ \\
ZTO50 & 8.3 & 20.2 & 0.78 & $5 \times 10^{8}$ \\
ZTO50-low & 9.6 & 9.8 & 2.5 & $5 \times 10^{5}$ \\
\hline
\end{tabular}

From the PDS absorption spectra in Figure 7, the Urbach energy for ZTO50-low is extracted to be $125 \mathrm{meV}$, which is a significant increase from the $102 \mathrm{meV}$ of ZTO50. This indicates that the density of defect states is higher with lower annealing temperatures. Therefore, there is a strong correlation between ZTO annealing temperatures and the Urbach energy. Moreover, the absorbance deep in the sub-bandgap is also higher in ZTO50-low than ZTO50, again showing strong correlation between ZTO annealing temperatures and deep defect states.

From our TFT and PDS data (Figures 4 to 7), it is observed that the effect of annealing temperatures on the ZTO TFT performance can be examined by the measured density of sub-bandgap states. This is consistent with a previous report on the use of PDS for organic devices [30]. On the other hand, the effect of tin compositions on the ZTO TFT performance does not show a strong correlation. However, the Urbach energy extracted is still valuable to gauge the quality of the semiconductors. 
Finally, the XRD and SEM analysis show that the ZTO33 and ZTO50 are very different crystalline structures and are also at different stages of crystallinity at a given annealing temperature. It can also be postulated that they will have different local short range order in their amorphous phases. This difference, which arises from different tin composition in the films, is in turn possibly related to the different field effect mobility obtained for the ZTO33 and ZTO50 TFTs. It is shown here that reactive sputtering achieves good devices from films with large window of zinc-to-tin ratio. This is consistent with wide process window of ZTO TFTs produced by other methods such as rf magnetron sputtering, pulse laser deposition and chemical vapour deposition [31-33]. One drawback of the reactive sputtering is the preferential sputtering of one metal over another, which would shorten the lifetime of such a target in a production environment.

4 Conclusions a-ZTO thin films have been deposited using remote-plasma reactive sputtering from zinc:tin alloy targets with various tin compositions. Optimised films are found to be stoichiometrically close to the common $\mathrm{Zn}_{2} \mathrm{SnO}_{4}$ and $\mathrm{ZnSnO}_{3}$ phases, which is confirmed by $\mathrm{XRD}$ and SEM on films annealed at various temperatures. At annealing temperatures $\sim 900{ }^{\circ} \mathrm{C}$, the $\mathrm{Zn}_{2} \mathrm{SnO}_{4}$ still remains stable but $\mathrm{ZnSnO}_{3}$ is decomposed to $\mathrm{Zn}_{2} \mathrm{SnO}_{4}$ and $\mathrm{SnO}_{2}$. TFTs incorporating these materials, after a postdeposition annealing at $500{ }^{\circ} \mathrm{C}$, exhibited good electrical characteristics with field effect mobilities $\sim 14$ to $20 \mathrm{~cm}^{2} \mathrm{~V}^{-}$ ${ }^{1} \mathrm{~s}^{-1}$. The field effect mobility increases with tin compositions but the Urbach energy does not vary with tin composition. On the other hand, the Urbach energy increases when the annealing temperature is reduced from 500 to $300{ }^{\circ} \mathrm{C}$, indicating that the defects in the bandgap increase. A field effect mobility $\sim 10 \mathrm{~cm}^{2} \mathrm{~V}^{-1} \mathrm{~s}^{-1}$ is still obtained in devices with $300{ }^{\circ} \mathrm{C}$ annealed ZTO, but there is a large shoulder at $V_{G S} \sim 0 \mathrm{~V}$ in the $I_{D S}-V_{G S}$ curve. The Urbach energies of the a-ZTO in this work are slightly lower than those previously reported for a-ZTO and a-IGZO. In summary it is shown that a-ZTO with very different tin compositions and microstructures are able to produce highly performing TFTs with mobilities in the same order.

Acknowledgements The support of this work by the Engineering and Physical Sciences Research Council (EPSRC) through project EP/M013650/1 is acknowledged. A.S. and R.H.F. would like to acknowledge funding and active support from EPSRC and India-UK APEX project. K.M.N. thanks Dr. S. Thornley of PlasmaQuest for providing the metallic tin target. Additional data related to this publication is available at the DSpace@Cambridge data repository (www.repository.cam.ac.uk).

\section{References}

[1] K. Nomura, H. Ohta, A. Takagi, T. Kamiya, M. Hirano, and H. Hosono, Nature 432, 488 (2004).
[2] H. Q. Chiang, J. F. Wager, R. L. Hoffman, J. Jeong, and D. A. Keszler, Appl. Phys. Lett. 86, 013503 (2005).

[3] M. Morales-Masis et al., Adv. Funct. Mater. 26, 384 (2016).

[4] Y. S. Lee, J. Heo, S. C. Siah, J. P. Mailoa, R. E. Brandt, S. B. Kim, R. G. Gordon, and T. Buonassisi, Energy Environ. Sci 6, 2112 (2013).

[5] S. Dutta and A. Dodabalapur, Sens. Actuators B 143, 50 (2009).

[6] J. A. Thornton and D. W. Hoffman, J. Vac. Sci. Technol. 14, 164 (1977).

[7] D. Hong, H. Q. Chiang, and J. F. Wager, J. Vac. Sci. Technol. B 24, L23 (2006).

[8] S. J. Wakeham, M. J. Thwaites, B. W. Holton, C. Tsakonas, W. M. Cranton, D. C. Koutsogeorgis, and R. Ranson, Thin Solid Films 518, 1355 (2009).

[9] W. B. Jackson and N. M. Amer, Phys. Rev. B 25, 25 (1982).

[10] R. Kelly and D. E. Harrison, Mater. Sci. Eng. 69, 449 (1985).

[11] K. Satoh, Y. Kakehi, A. Okamoto, S. Murakami, F. Uratani, and T. Yotsuya, Jpn. J. Appl. Phys. 44, L34 (2005).

[12] D. L. Young, H. Moutinho, Y. Yan, and T. J. Coutts, J. Appl. Phys. 92, 310 (2002).

[13] J. S. Rajachidambaram, S. Sanghavi, P. Nachimuthu, V. Shutthanandan, T. Varga, B. Flynn, S. Thevuthasan, and G. S. Herman, J. Mater. Res., 27, 2309 (2012).

[14] I. Stambolova, K. Konstantinov, D. Kovacheva, P. Peshev, and T. Donchev, J. Solid State Chemistry 128, 305 (1997).

[15] Y.-S. Shen and T.-S. Zhang, Sens. Actuators B 12, 5 (1993).

[16] Y. Hayashi, K. Kondo, K. Murai, T. Moriga, I. Nakabayashi, H. Fukumoto, and K. Tominaga, Vacuum 74, 607 (2004).

[17] J. J. Robbins, Y.-J. Huang, M. Bai, T. L. Vincent, and C. A. Wolden, Mat. Res. Soc. Symp. Proc. 666, F1.7.1 (2001).

[18] C. Tsakonas, W. Cranton, F. Li, K. Abusabee, A. Flewitt, D. Koutsogeorgis, and R. Ranson, Journal of Physics D: Applied Physics 46, 095305 (2013).

[19] N. Nikolic, T. Sreckovic, and M. M. Ristic, Journal of the European Ceramic Society 21, 2071 (2001).

[20] Y.-C. Ji, H.-X. Zhang, X.-H. Zhang, and Z.-Q. Li, Physica status solidi (b) 250, 2145 (2013).

[21] A. J. Flewitt et al., Semicond. Sci. Technol. 24, 085002 (2009).

[22] T. Kamiya and H. Hosono, NPG Asia Materials 2, 15 (2010).

[23] N. Mitoma, S. Aikawa, X. Gao, T. Kizu, M. Shimizu, M.-F. Lin, T. Nabatame, and K. Tsukagoshi, Appl. Phys. Lett. 104, 102103 (2014).

[24] J. Robertson, Journal of Non-Crystalline Solids 358, 2437 (2012).

[25] A. Sadhanala et al., J Phys Chem Lett 5, 2501 (2014). 
[26] R. L. Hoye, S. Heffernan, Y. Ievskaya, A. Sadhanala, A. Flewitt, R. H. Friend, J. L. MacManus-Driscoll, and K. P. Musselman, ACS applied materials \& interfaces 6, 22192 (2014). [27] P. T. Erslev, E. S. Sundholm, R. E. Presley, D. Hong, J. F. Wager, and J. D. Cohen, Applied Physics Letters 95, 192115 (2009).

[28] E. K.-H. Yu, S. Jun, D. H. Kim, and J. Kanicki, Journal of Applied Physics 116, 154505 (2014).

[29] K. Nomura, T. Kamiya, H. Yanagi, E. Ikenaga, K. Yang, K. Kobayashi, M. Hirano, and H. Hosono, Applied Physics Letters 92, 202117 (2008).

[30] J. Socratous, K. K. Banger, Y. Vaynzof, A. Sadhanala, A. D. Brown, A. Sepe, U. Steiner, and H. Sirringhaus, Adv Funct Mater 25, 1873 (2015).

[31] R. L. Hoffman, Solid-State Electronics 50, 784 (2006).

[32] P. Görrn, P. Hölzer, T. Riedl, W. Kowalsky, J. Wang, T. Weimann, P. Hinze, and S. Kipp, Appl. Phys. Lett. 90, 063502 (2007).

[33] U. K. Kim et al., J. Mater. Chem. C 1, 6695 (2013). 